\title{
Development of a Cognitive Assessment Tool and Training Systems for EIderly Cognitive Impairment
}

\author{
A case study of a successful development of a cognitive assessment tool and training \\ systems for the elderly people in South Korea
}

\author{
Gyu Hyun Kwon, Laehyun Kim, and Sehyung Park \\ Center for Bionics \\ Korea Institute of Science and Technology \\ Seoul, Republic of Korea \\ \{ghkwon, laehyunk, sehyung\}@kist.re.kr
}

\begin{abstract}
The authors introduce a computer-based cognitive assessment tool and two different types of training systems. CoCoMO is a new computer based cognitive assessment tool for middle-aged and old people in South Korea. CoCoTA is a computer-based cognitive training tool considering cultural aspects. E-CoRe is a new cognitive training platform in order to reinforce the coupling of human body and cognition. E-CoRe was built with a tangible tabletop interface with real tangible objects and computerized table. These cognitive rehabilitation systems were aimed to detect cognitive impairment in early time and improve cognitive function and gross motor skills of older adults by providing daily life training and by interacting with real tangible objects.
\end{abstract}

Keywords-Cognitive Assessment; Cognitive Training; Mild Cognitive Impairment; Cognitive Rehabilitation

\section{Introduction}

With the aging population, the number of middle-aged and old people with cognitive impairments is rapidly increasing in Korea. One out of ten among people aged 65 and older in Korea are suspected of Alzheimer's disease. The problem is so grave that the social cost for Alzheimer's disease is expected to reach approximately 200 billion USD by the year 2020 .

There are some evidences that cognitive training could improve cognitive function, which potentially slows cognitive decline and prevent the age-related cognitive problem such as dementia [1, 2]. The earlier a cognitive impairment is diagnosed, the better the prospect that it can be prevented. The first visit to hospitals of Korean patients with dementia is average 2.7 year after the first symptom was identified. On the other hand, it takes only 1.4 year at developed countries [3]. However, the K-MMSE, a translated version of the minimental state examination (MMSE), does not meet enough sensitivity to screening mild cognitive impairment (MCI). In addition, The Seoul neuropsychological screening battery (SNSB), a sophisticated screening tool developed in South Korea, was expensive and too laborious [4]. Thus, there is strong need to develop a screening tool with high sensitivity yet simple. Furthermore, even though there are several computer-based cognitive training tools in South Korea, most of them are not clinically validated. Well-designed cognitive training systems in other countries such as Lumosity ${ }^{\mathrm{TM}}$ are not culturally compatible to the elderly people in South Korea.
Additionally, the legacy computer interface such as a mouse and a keyboard is still a big barrier for the elderly people. A series of the previous study showed tangible object and multitouch tabletop computer could be one of the best options of more accessible computing for the elderly people [5-7].

Thus, the center for Bionics at the Korea Institute of Science and Technology has worked on a computerized measurement tool for early detection of cognitive impairment and two training systems for improving cognitive ability in partnership with Seoul National University Hospital at Bundang, Hallym University, Primpo Co., Ltd, and Flow Gamez.

In this paper, we will briefly introduce three different systems to prevent cognitive decline for MCI in South Korea. Through the workshop, we expect to extensively share our experiences we gained throughout the development of the systems along with the problems we confronted, in order to support healthy and active aging.

\section{Systems}

\section{A. $\mathrm{CoCoMO}$}

Computerized Cognitive Assessment in Middle Aged and Older Adults (CoCoMO) is a PC-based cognitive ability measurement tool. It can perform 13 assignments in 7 cognitive areas. It measures cognitive ability by testing functions that are closely related to daily living such as basic numeration, counting money, and recalling words or locations. Backed by Norm Data Base with the sample size of 745 people, CoCoMO is a rigorously tested evaluation module with a high degree of validity and reliability. It is the first of its kind in South Korea. CoCoMO can test mild cognitive impairment in early stages $[8,9]$. CoCoMO can significantly save time and costs over the conventional administration of the paper pencil test and a follow-up brain tomography.

CoCoMO enables focused measurement. It is designed to minimize incidental hindrance associated with measurements. With CoCoMO, anyone with basic computer skills can measure their cognitive ability. Currently plans to provide trial services are underway at medical service providers such as hospitals, community health care centers, and health examination centers across the country. 


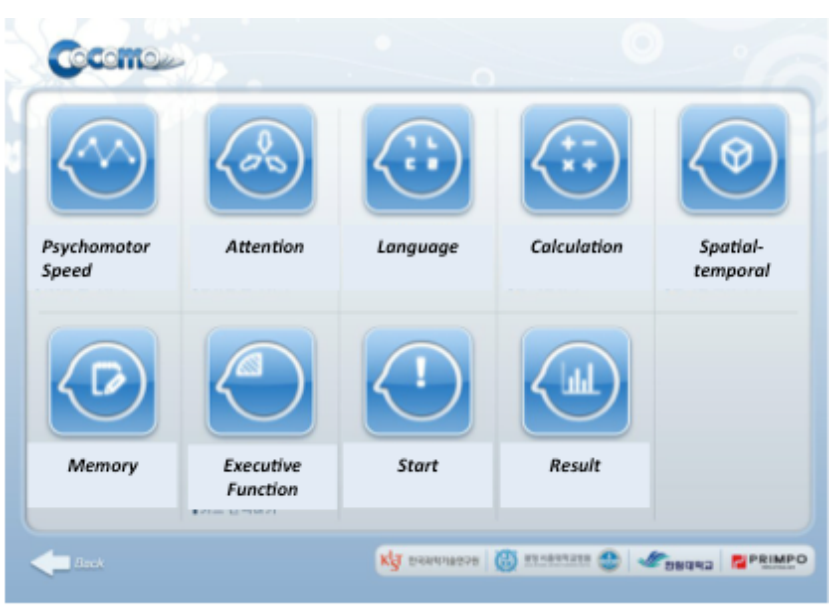

Fig. 1. A Screenshot of CoCoMO (Computerized Cognitive Assessment in Middle Aged and Older Adults)

\section{B. $\operatorname{CoCoTA}$}

Computerized Cognitive Training Apparatus (CoCoTA) is a cognition-training program to improve and maintain cognitive function for the middle-aged and old people. It is basically a self-training online program for people with MCI and for normal people as well, which records the history of the training and manages the cognitive health of a user. It consists of 14 cognitive training tools.

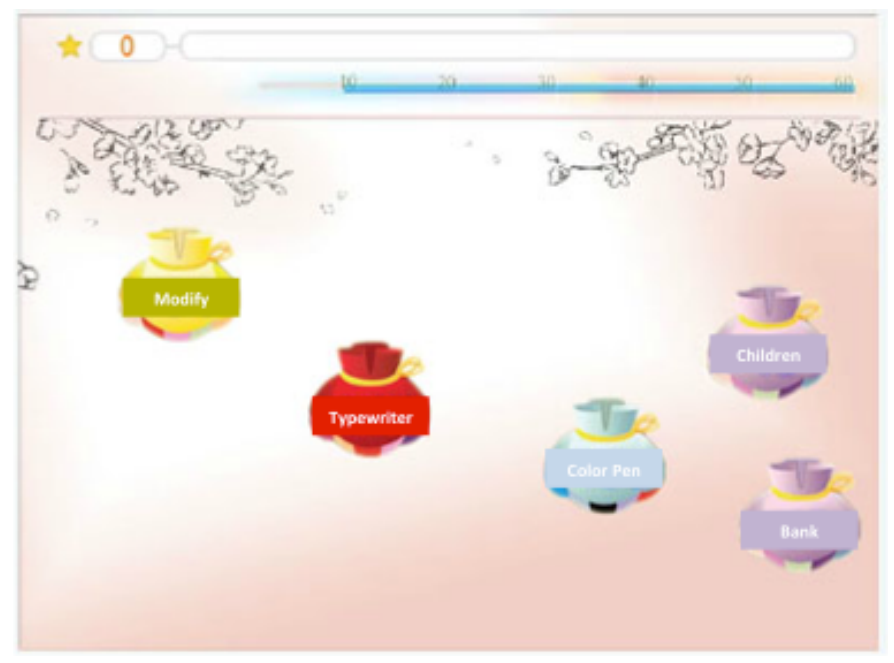

Fig. 2. A Screenshot of "find a word" training component *

"Find a Word" is a game to find a word with spelling containing a letter found in the pocket. It is designed to engage diverse cognitive areas related to executive functions and concentration. Games such as "Remembering Traditional Playing Cards" and "Find a Brass Coin" were developed with

\footnotetext{
* Original version was written in Korean. For readability, we overlaid English meaning in each Korean word respectively.
}

awareness toward our cultural background and familiarity for the elderly. CoCoTA is designed with visually stimulating colors and shapes for the elderly. Providing clear and quick responses, CoCoTA's game-like makeup is designed to attract testers' attention. Based on scientific clinical theory, CoCoTA is a game developed to enable objective measurement and evaluation of cognitive training. At present, our researchers are conducting clinical trials by observing the changes in brain function before and after conducting cognitive training using CoCoTA.

\section{E-CoRe}

E-CoRe System goes beyond the existing cognitive training methods that work only on specific cognitive areas $[10,11]$. As a cognitive rehabilitation training system based on embodied cognition theory, E-CoRe System makes simultaneous training of cognition and physical movement possible. We built a table-top based cognitive training platform in E-CoRe System. Making use of actual tools in daily living, E-CoRe System is designed with intuitive tangible interfaces for the elderly to operate easily $[11,12]$.

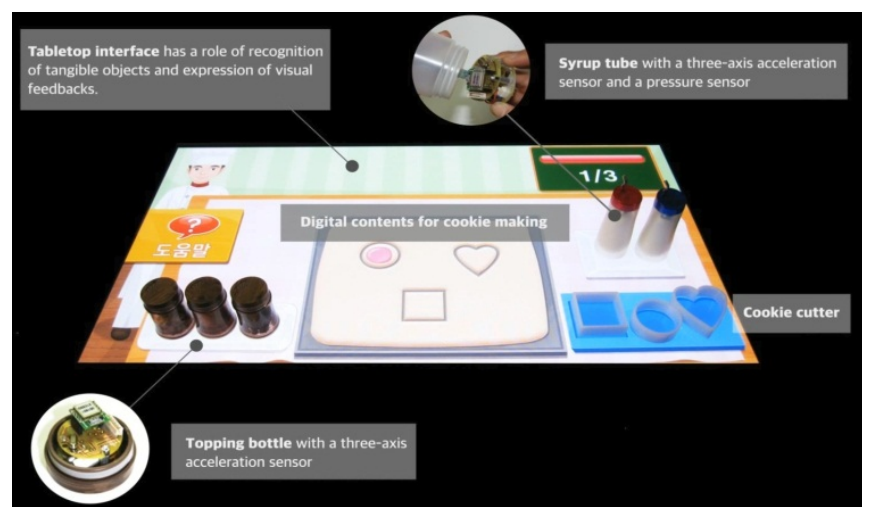

Fig. 3. Overview of E-CoRe (Embodied COgnitive REhabilitation) system

"Making Cookies" aims to improve three cognitive areas: memory, executive function and attention. The baking stage requires adjusting the number, shapes of cookies, and the oven temperature, while the finishing-touch stage asks testers to add syrup and toppings. Each step proceeds according to systematically assigned difficulty levels based on scientific training theory. Four execution tools (syrup, cookies, topping and stamps) are embedded with a triaxis acceleration censor, a 1-channel barometric censor, and a Bluetooth wireless communication module. It operates on a multi-touch tabletop display, coupled with object location and identification system. E-CoRe System's modular design makes training of the whole or partial activities of daily living (ADL) possible. Its computer-based system can measure and evaluate results of cognitive training objectively. Lauded as a cognitive training tool fit for the middle aged and the elderly, E-CoRe System opened up a new possibility in the cognitive training paradigm with its adoption of an easy-to-use, intuitive interactive interface with increased response speed. It is also recognized as a new model for the cognition management platform with online-offline integration. 


\section{Conclusion}

In this paper, we presented one cognitive assessment tool, one computer based cognitive training tool, and a new interface based cognitive training platform. CoCoMO and CoCoTA are effective systems for cognitive assessment and training using conventional technology. E-CoRe is a new attempt to apply TTI-based ADL tasks to cognitive training so as to promote interconnections between human behavior and cognitive processes. CoCoMO finished clinical test at two different hospitals, which was reported at several Korean conferences [8, 9]. We are preparing the deployment of CoCoMO at the public health service in South Korea. CoCoTA just finished clinical validation at both the Bobath Memorial Hospital and Seoul National University Hospital at Bundang. It is ready to be reported. E-CoRe was reviewed by clinical and system experts [13]. In cooperation with a major hospital at Seoul, we are just starting the user study for ECoRe system. We believe that our systems represent a new interactive therapeutic approach toward better cognitive rehabilitation.

\section{Acknowledgment}

This work has been supported by the Assessment Technology of Cognitive Ability for Elderly People Sponsored by Ministry of Knowledge Economy and partly supported by the Future Fundamental Program of Korea Institute of Science and Technology [Image-Guided Surgical System].

\section{References}

[1] S. Belleville, "Cognitive training for persons with mild cognitive impairment," Int Psychogeriatr, vol. 20, Feb 2008, pp. 57-66.

[2] V. Faucounau, Y. H. Wu, M. Boulay, J. De Rotrou, and A. S. Rigaud, "Cognitive Intervention Programmes on Patients Affected by Mild Cognitive Impairment: A Promising Intervention Tool for MCI?,"

[3] N. Park, "9\% of the population over 65 years old have cognitive impairment", Chosun il-bo(Sep. 08, 2010), Section. Health

[4] H.J. Ahn, J. Chin, A. Park, B.H. Lee, M.K. Suh, S.W. Seo, D.L. Na, "Seoul Neuropsychological Screening Battery-Dementia Version (SNSB-D): A Useful Tool for Assessing and Monitoring Cognitive Impairments in Dementia Patients", Journal of Korean Medical Science, v.25(7), Jul 2010, pp. 1071-1076

[5] K. Sitdhisanguan, N. Chotikakamthorn, A. Dechaboon, and P. Out, "Using tangible user interfaces in computer-based training systems for low-functioning autistic children," Personal and Ubiquitous Computing, vol. 16, Feb 2012, pp. 143-155.

[6] L. Gamberini, F. Martino, B. Seraglia, A. Spagnolli, M. Fabregat, F. Ibanez, M. Alcaniz, and J. M. Andres, "Eldergames project: An innovative mixed reality table-top solution to preserve cognitive functions in elderly people," in Human System Interactions, 2009. HSI '09. 2nd Conference on, 2009, pp. 164-169.

[7] T. Marques, F. Nunes, P. Silva, and R. Rodrigues, "Tangible Interaction on Tabletops for Elderly People," in Entertainment Computing - ICEC 2011. vol. 6972, J. Anacleto, S. Fels, N. Graham, B. Kapralos, M. Saif El-Nasr, and K. Stanley, Eds., ed: Springer Berlin Heidelberg, 2011, pp. 440-443.

[8] M. Song, J. Park, Y. Kang, K. H. Yu, and B. C. Lee "A comparison of the performance on the Korean-montreal cognitive assessment (KMoCA) between amnestic MCI and dysexecutive MCI" Korean Dementia Spring Conference, Apr. 2011.

[9] Y. Kang, M. Song, J. Park, K. H. Yu, and B. C. Lee "An exploration of subgroups in vascular cognitive impairment no dementia (VCIND)" The 39th Annual Meeting of the International Neuropsychological Society, Feb. 2011, Boston.

[10] D. E. Barnes, K. Yaffe, N. Belfor, W. J. Jagust, C. DeCarli, B. R. Reed, and J. H. Kramer, "Computer-based cognitive training for mild cognitive impairment: results from a pilot randomized, controlled trial," Alzheimer Dis Assoc Disord, vol. 23, Jul-Sep 2009, pp. 205-10.

[11] C. Herrera, C. Chambon, B. F. Michel, V. Paban, and B. AlescioLautier, "Positive effects of computer-based cognitive training in adults with mild cognitive impairment," Neuropsychologia, Apr. 2012.

[12] G. Kim, K. Song, G. H. Kwon, and L. Kim, "A Cognitive Rehabilitation Content for the Tangible Table-Top Interface" Korean HCI Conference, Jan. 2012, Pyungchang, Korea.

[13] J. Jung, L. Kim, S. Park, and G. H. Kwon, " E-CORE (Embodied COgnitive REhabilitation): A Cognitive Rehabilitation System Using Tangible Tabletop Interface" Int. Conf. Neuro-Rehabilitation, Nov. 2012, Toledo, Spain. 